\title{
A!
}

This is an electronic reprint of the original article.

This reprint may differ from the original in pagination and typographic detail.

Saleh, Taha; Petrov, Dmitry; Tirkkonen, Olav; Räisänen, Vilho

\section{Probabilistic Path Loss Predictors for mmWave Networks}

\section{Published in:}

Proceedings of IEEE 93rd Vehicular Technology Conference, VTC 2021

DOI:

10.1109/VTC2021-Spring51267.2021.9448967

Published: 15/06/2021

Document Version

Peer reviewed version

Please cite the original version:

Saleh, T., Petrov, D., Tirkkonen, O., \& Räisänen, V. (2021). Probabilistic Path Loss Predictors for mmWave Networks. In Proceedings of IEEE 93rd Vehicular Technology Conference, VTC 2021 [9448967] (IEEE

Vehicular Technology Conference; Vol. 2021-April). IEEE. https://doi.org/10.1109/VTC2021-

Spring51267.2021.9448967

This material is protected by copyright and other intellectual property rights, and duplication or sale of all or part of any of the repository collections is not permitted, except that material may be duplicated by you for your research use or educational purposes in electronic or print form. You must obtain permission for any other use. Electronic or print copies may not be offered, whether for sale or otherwise to anyone who is not an authorised user. 
(C) 2021 IEEE. This is the author's version of an article that has been published by IEEE. Personal use of this material is permitted. Permission from IEEE must be obtained for all other uses, in any current or future media, including reprinting/republishing this material for advertising or promotional purposes, creating new collective works, for resale or redistribution to servers or lists, or reuse of any copyrighted component of this work in other works. 


\title{
Probabilistic Path Loss Predictors for mmWave Networks
}

\author{
Taha Saleh* ${ }^{* \dagger}$, Dmitry Petrov ${ }^{\dagger}$, Olav Tirkkonen*, Vilho Räisänen ${ }^{\dagger}$ \\ *Aalto University, Espoo, Finland \{first.last $@ @$ aalto.fi \\ ${ }^{\dagger}$ Nokia Bell Labs, Espoo, Finland \{dmitry.a.petrov, vilho.raisanen\}@ nokia-bell-labs.com
}

\begin{abstract}
End-to-end network performance evaluation and dynamic resource provisioning require models that are fast in execution and produce predictions in a probabilistic way, including accuracy estimations. mmWave mobile networks are challenging for the analysis due to the difference in line of sight $(\mathrm{LoS})$ and non-line of sight $(\mathrm{NLOS})$ regimes. The training and accuracy of the models depend on the amount of available measurement data and domain knowledge. In this paper, we consider two probabilistic models for path loss prediction in mmWave networks. Both, a Bayesian learning and a Mixture Density neural Network (MDN) models are developed and trained to predict path loss distributions in a realistic city environment based on a limited amount of training data. We measure prediction capability in terms of Kullback-Leibler (KL) divergence and Total Variation Distance (TVD). The results show that MDN describes path loss more accurately for larger training data-sets. However, the Bayesian learning predictor is more data-efficient.

Index Terms-Bayesian learning, path loss, mmWave, mixture density networks
\end{abstract}

\section{INTRODUCTION}

$5 \mathrm{G}$ is the enabler of digital services with very different performance characteristics, including (ultra) low latency, (ultra) high reliability, (ultra) wide bandwidth. A key enabler for diverse applications is network slicing, which allows for the provision of differentiated end-to-end services for users. The end-to-end service needs to be mapped to domain-specific components, e.g., Radio Access Network (RAN) slices. ETSI Zero-Touch Network and Service Management (ZSM) [1] provides a framework for mapping end-to-end service provisioning to domain components in multi-vendor operator environments.

Design and orchestration of new slices and dynamic service quality assurance is a complicated task, especially for wireless mobile networks that are stochastic by their nature. It is hard to model their behavior within a limited time-frame. Therefore, sophisticated but computationally feasible models are needed to evaluate possible values of key performance indicators (KPI) that need to be controlled according to the service level agreement (SLA) of the slice.

One way to tackle this problem is to run detailed simulations that take multiple factors and features into account. This approach has proven its efficiency when there is sufficient time for such simulations and enough of computational resources, e.g., at the network planning stage or the development of algorithms.

A computationally more efficient alternative is to use analytical models to predict network performance [2]. Analyt- ical approaches can be based on fluid [3], flow-based [4] and stochastic geometry models [5]. Most of them are well applicable for the analysis of generic scenarios and relative comparison of the results. However, getting correct absolute values of KPIs in concrete environments and network deployments is still challenging. Moreover, with these methods, it is usually possible to get only average values of KPIs, whereas some SLA requirements are set as maximum (e.g., latency) or minimum (e.g., throughput).

Thus, we study a hybrid approach to build machine learning (ML) models that can describe network KPIs in the form of probability distributions fitted for a concrete scenario. The parameters of the predictors are defined as random variables. The distributions of the parameters are inferred from data collected either from a real network or from a detailed simulator. We start the analysis of such an approach with the predictors describing distance-dependent path loss (PL) in mmWave 5G networks deployed in a realistic city environment. In the future, we plan that a similar approach can be extended to the other network KPIs such as signal to interference plus noise ratio, throughput, and latency.

In this paper, we describe two types of probabilistic path loss predictors. The first method uses Bayesian learning to get the posterior distribution of the parameters of an analytical model and to make predictive inference of path loss values. In the second approach, a probabilistic neural network is implemented to get the parameters of analytical distributions.

Collection of training data is a costly operation. It might be additionally complicated by the need to know accurate coordinates of the measurements. Hence, we study how the path loss predictors perform given the limited size of training dataset. Two metrics are proposed for the comparison. Moreover, we also examine if the predictors can be trained for individual sites and if this can result in better prediction quality. Note that the focus of the paper is to investigate the predictors' performance for the particular environment from which the data-set is obtained.

The rest of the paper is arranged as follows. In the next section, we present the simulations environment in more detail and overview existing approaches to evaluate path loss in mmWave bands. In Section III, Bayesian Learning (BL) and Neural Network (NN) based path loss predictors are described. In Section IV, we introduce several metrics and compare the models according to them. Finally, we conclude the paper in Section V. 


\section{Propagation ENVIRONMENT AND PATH LOSS MODELS}

mmWave carriers typically in the $24-52 \mathrm{GHz}$ frequency range were introduced in $5 \mathrm{G}$ to get additional spectrum resources. These new bands can cover $800 \mathrm{MHz}$ or even wider continuous spectrum intervals and support lower latency due to shorter transmission time intervals. On the downside are more substantial signal attenuation that can be compensated by the use of radios with massive multiple-input and multiple-output (mMIMO) antennas, and higher exposure to shadowing and blocking. The letter reduces predictability of wireless channels in non-line of sight $(\mathrm{NLoS})$ regime.

Collecting a sufficiently large data-set of field measurements for fitting of models and training of predictors is a tedious task. In our work, we analyze the impact of the amount of available data on the quality of path loss predictions. We use the path loss results from a commercial specialised ray-tracing tool as a reasonably close alternative to real measurements. The input for 3D ray-tracing calculations at $28 \mathrm{GHz}$ carrier with $2 \mathrm{~m}$ resolution was accurate $3 \mathrm{D}$ building data from a city in the United States.

The scenario includes eight mmWave Base Station (BS) sights at the crossroads of an almost uniform city grid with inter-site distances around $110 \mathrm{~m}$ in vertical direction and 200 $\mathrm{m}$ in horizontal. 3D multi-path ray-tracing simulations were done for each site covering the whole area of roughly 400 $\times 500$ square meters in size. The height of the BS and user equipment (UE) antenna was selected to be $10 \mathrm{~m}$ and $1.5 \mathrm{~m}$, correspondingly.

Path loss data was collected from 100 independent simulator runs. With this procedure, we model the creation of snapshots from the real network with 12 UEs connected to each BS site on average, i.e., 96 UEs per simulation result in total. All UEs were placed randomly only in outdoor locations. As a result, we get a 2D probabilistic distribution of UE-BS distances and path loss values shown in Figure 1 for the whole scenario. Random sub-samples of different sizes from this large dataset are then used for the training and verification of path loss predictors. Additionally, we analyze path loss data-sets for each of the BS sites individually.

\section{A. Path loss models}

A thorough overview of parametric channel models for $5 \mathrm{G}$ can be found in [6] by Rappaport et.al. In [7], Close-In model with a Frequency-weighted path loss exponent (CI) and 1 $\mathrm{m}$ free-space reference provides the same level of prediction accuracy but greater parameter stability as compared to other similar parametric formulae. Therefore, in our study the following general form of CI is used as a reference:

$$
\mathrm{PL}[\mathrm{dB}]=10 n \log _{10} d_{3 D}+32.4+20 n \log _{10} f+\chi_{\sigma} .
$$

Here, $d_{3 D}$ is the 3D BS-UE separation distance in meters, $n$ denotes the path loss exponent, $f$ is the carrier frequency in $\mathrm{GHz}, \chi_{\sigma}$ is a zero-mean Gaussian random variable with standard deviation $\sigma$ in $\mathrm{dB}$ modeling shadow fading. The close-in free-space distance is $1 \mathrm{~m}$.

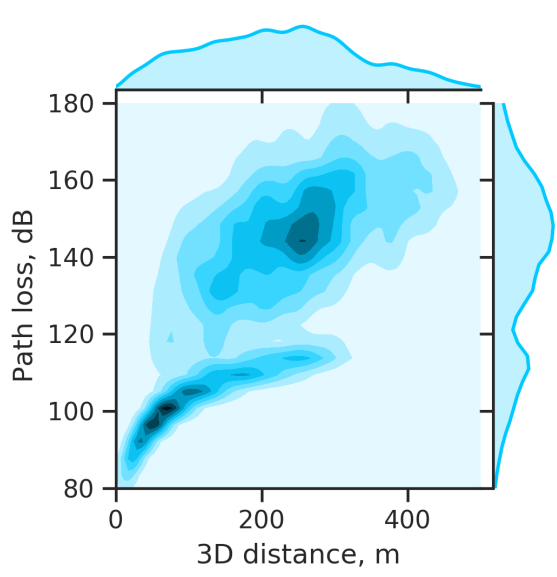

Fig. 1. Joint distribution of UE-BS distances and path losses for the entire scenario.

Several types of LoS probability models can be found in literature [8]. We adopt here one also used in 3GPP [9]:

$$
p_{\mathrm{LoS}}\left(d_{2 D}\right)=\left[\min \left(\frac{d_{0}}{d_{2 D}}, 1\right)\left(1-e^{-\frac{d_{2 D}}{d_{1}}}\right)+e^{-\frac{d_{2 D}}{d_{1}}}\right] \text {, }
$$

where $d_{2 D}$ is the 2D BS-UE distance in meters, $d_{0}$ is the break-point distance below which the LoS probability stays equal to 1 , and $d_{1}$ is a parameter describing the power of decay.

To improve modeling accuracy, path loss models utilizing Machine Learning (ML) techniques have been explored lately. In such path loss models, measurement data is used to learn the mapping function between features and path loss. In [10], the authors have utilized Support Vector Machines (SVM) for the prediction of the path loss. System-specific parameters (antenna separation distance, horizontal and vertical antenna attenuation) are used along with environment-specific parameters (longitude, latitude, and terrain elevation) as input features of the ML model. Similarly, in [11], a propagation model is implemented using a deep learning approach. The terrain profile of the environment, along with antenna separation distance, are used for making predictions. Both [10] and [11] have shown that ML-based models perform better compared to the analytical ones.

ML methods depend on the type of information that is used for training. If feature engineering is applied, the input features are carefully selected utilizing the domain knowledge. Both [10] and [11] have utilized this approach by extracting the information of the environment in which the model is being deployed. This requires extra effort instead of just using raw antenna-separation distance as an input feature. In this paper, both the models have utilized only antenna-separation distance to predict path loss. Furthermore, domain knowledge can also be embedded in the ML model in the form of analytical equations. The BL model presented in this works take benefits of this approach.

Moreover, ML-based path loss models deals with point estimation of the path loss. As a result, uncertainty in the 
predicted values is not taken into consideration. In this study, we construct the predictors that provide distributions as an output, and hence, take uncertainty into account. Furthermore, the amount of training data has an impact on the prediction quality. Models necessitating a higher quantity of training data would require more field measurements to be carried out, which would, in turn, incur higher expenses. To the best of our knowledge, the impact of the amount of training data on the ML-based path loss models is not investigated in the literature.

\section{PROBABILISTIC PATH LOSS MODELS}

In this work, we consider two new models for path loss prediction. The first model uses a probabilistic ML approach on top of an analytical parametric model, which takes domain knowledge into account. The second model employs an artificial NN structure. Both ML models, instead of providing point estimates, aim at learning the underlying joint distribution of path loss and distance. Additionally, we always assume that mmWave propagation has two different regimes: LoS and NLoS.

\section{A. PL model based on Bayesian learning}

The core idea of Bayesian learning is to use the posterior distribution of model parameters for predictive inference, i.e., to build the probability distribution for a new unobserved data point. The posterior distribution of parameters $\boldsymbol{\theta}$ for data points $x \in X$ with distribution $x \sim p(x \mid \boldsymbol{\theta})$ is given by the Bayes theorem:

$$
p(\boldsymbol{\theta} \mid X, \boldsymbol{\alpha})=\frac{p(X \mid \boldsymbol{\theta}, \boldsymbol{\alpha}) p(\boldsymbol{\theta} \mid \boldsymbol{\alpha})}{p(X \mid \boldsymbol{\alpha})},
$$

where $X$ is the data-set containing the results of observations and $\boldsymbol{\alpha}$ are observable input data and hyper-parameters of parameter distributions $\boldsymbol{\theta} \sim p(\boldsymbol{\theta} \mid \boldsymbol{\alpha})$. Then, a Bayesian prediction for a new data point $\tilde{x}$ is

$$
p(\tilde{x} \mid X, \boldsymbol{\alpha})=\int p(\tilde{x} \mid \boldsymbol{\theta}) p(\boldsymbol{\theta} \mid X, \boldsymbol{\alpha}) d \boldsymbol{\theta}
$$

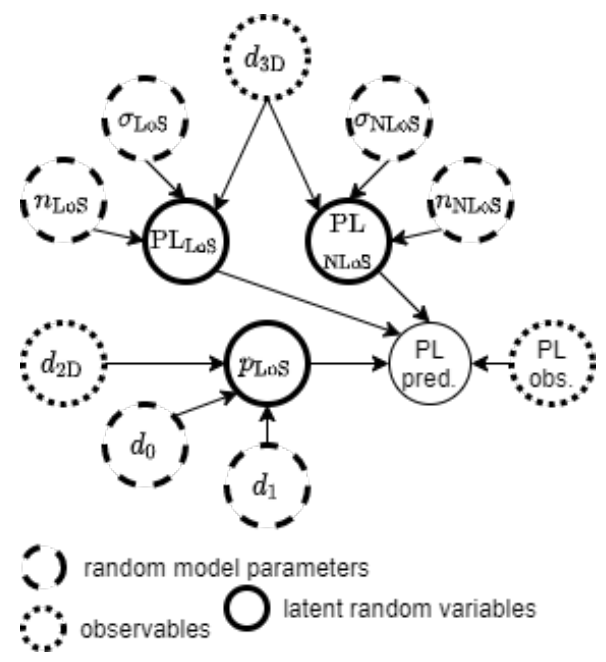

Fig. 2. The scheme of probabilistic path loss (PL) Bayesian model.
TABLE I

BAYESIAN PATH LOSS MODEL PRIORS AND POSTERIORS

\begin{tabular}{|c|c|c|}
\hline Model parameter & Prior distribution & Distribution parameters \\
\hline \hline$n_{\mathrm{LOS}}$ & Normal $(\mu, \sigma)$ & $\mu=2.9, \sigma=10.0$ \\
\hline$n_{\mathrm{NLOS}}$ & Normal $(\mu, \sigma)$ & $\mu=4.0, \sigma=10.0$ \\
\hline$\sigma_{\mathrm{LOS}}$ & HalfNormal $(\sigma)$ & $\sigma=10.0$ \\
\hline$\sigma_{\mathrm{NLOS}}$ & HalfNormal $(\sigma)$ & $\sigma=20.0$ \\
\hline$d_{0}$ & Gamma $(\alpha, \beta)$ & $\alpha=24.2, \beta=1.1$ \\
\hline$d_{1}$ & Gamma $(\alpha, \beta)$ & $\alpha=163.3, \beta=2.3$ \\
\hline
\end{tabular}

For the purpose of path loss modelling we build a Bayesian model as summarized in Fig. 2:

- The input data $\boldsymbol{\alpha}$ are 3D and 2D TR distances $d_{3 D}$ and $d_{2 D}$. Carrier frequency $f$ is fixed and equal to $28 \mathrm{GHz}$.

- The parameters $\boldsymbol{\theta}$ are the path loss exponents $n_{\mathrm{LoS}}$, $n_{\mathrm{NLoS}}$ and standard deviations $\sigma_{\mathrm{LoS}}, \sigma_{\mathrm{NLoS}}$ from (1). Two additional parameters are $d_{0}$ and $d_{1}$ from (2).

- The likelihoods of path loss values $x \in X$ in (3) are given by a probabilistic mixture of LoS and NLoS path loss probabilities [12]:

$$
p(\mathrm{PL})=p_{\mathrm{LoS}} p\left(\mathrm{PL}_{\mathrm{Los}}\right)+\left(1-p_{\mathrm{LoS}}\right) p\left(\mathrm{PL}_{\mathrm{NLoS}}\right),
$$

where we assume that LoS and NLoS path loss distributions $p\left(\mathrm{PL}_{\mathrm{Los}}\right)$ and $p\left(\mathrm{PL}_{\mathrm{NLoS}}\right)$ are normal with mean and standard deviations defined by the random parameters $n_{\mathrm{LoS}}, n_{\mathrm{NLoS}}$ and $\sigma_{\mathrm{LoS}}, \sigma_{\mathrm{NLOS}}$ substituted into expression (1). LoS probability $p_{\mathrm{LoS}}$ is defined by (2) with random parameters $d_{0}$ and $d_{1}$.

- Finally, the priors $p(\boldsymbol{\theta} \mid \boldsymbol{\alpha})$ in (3) contain additional information available about the model parameters. The choice of prior distributions might have considerable impact on the model accuracy and training convergence. We use 3GPP model parameters [9] for LoS/NLoS path loss as a reference. The types of prior distributions and their parameters are summarized in Table I.

In practice, the denominator in (3) might not be tractable. Moreover, analytical derivation of posterior distributions in most of the real cases is a complicated task. For these reasons, Markov Chain Monte Carlo (MCMC) [13] computational method is often used for Bayesian inference, and is applied in this paper. MCMC allows drawing samples from a distribution even if it cannot be derived analytically. Hence, it becomes possible to get the approximate posterior distributions, like the one shown in Figure 3 for parameter $\sigma_{\mathrm{NLoS}}$. Depending on the size of the training data-set, we used from $80 \mathrm{~K}$ to $120 \mathrm{~K}$ steps in the MCMC procedure, while leaving out between $10 \mathrm{~K}$ and $20 \mathrm{~K}$ first steps for the burn-in.

The implementation of the model and training algorithm was done in Python with TensorFlow Probability (TFP) [14]. This library for probabilistic reasoning and statistical analysis was recently introduced as a part of TensorFlow ML platform. Besides a rich tool-set of different distributions, it also provides the implementation of probabilistic, variational, and optimization algorithms. In particular, we used the Hamiltonian version of MCMC for the efficient sampling in 


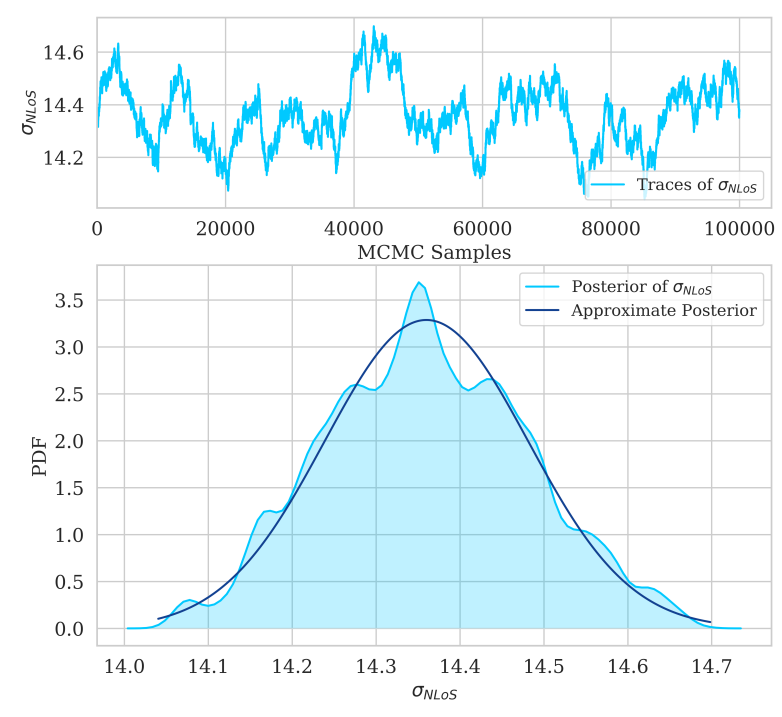

Fig. 3. Traces (top) and posterior (bottom) of path loss model parameter $\sigma_{\mathrm{NLOS}}$.

the six-dimensional parameter space of the path loss model. Additionally, TFP can be easily integrated with deep learning models which can be useful in chaining multiple ML-based models for end-to-end network performance prediction.

For the convergence of the model, the value of hyperparameters is crucial. The hyper-parameters includes the parameters of the prior distributions and the step size of MCMC. In total, there are 11 hyper-parameters. The values of these hyper-parameters were set to provide faster optimization. For hyper-parameter of prior distributions, the values were selected as per 3GPP recommendation. These values are presented in Table I. In the case of step size, the value is initially set to 0.5 which is then updated by the adaptive algorithm given in [15].

For training the model a portion of the data-set was used. To check the model accuracy posterior predictive check [16] was performed. Different distances were considered for which posterior distribution was obtained. The predicted distribution was then compared to the true PL distribution.

As a result of Bayesian inference, we solve a probabilistic non-linear classification problem between $\operatorname{LoS}$ and NLoS propagation. However, one of the crucial benefits of the model is that it does not require the knowledge of LoS/NLoS conditions of the UEs. This information is hardly available outside of special measurement campaigns. Hence, LoS probability $p_{\text {LoS }}$ is the latent variable inferred at the predictor training phase.

\section{B. PL model based on NN}

The second predictor that we are building is a Mixture Density Network (MDN), which is also capable to provide a distribution of path losses instead of point estimates. MDN was firstly introduced by C.M. Bishop in [17]. It is a combination of conventional Neural Network (NN) and a mixture model. The NN contains a certain number of processing elements called nodes, which are densely connected and arranged in layers. They have the capability of representing any arbitrary function [18]. As a result, one of the benefits of using an NNbased algorithm is that it is not necessary to assume any close form expression for path loss and LoS probability formulae, in contrast to the Bayesian predictor.

Moreover, a mixture component aims at representing the true distribution with a mixture of distributions [18]. The distributions have weights assigned to them. These probabilistic weights specify the contribution of each distribution in the mixture. In addition to weights, there are also other parameters that characterize the distributions. For example, if a Gaussian family of distributions is used, the parameters of the mixture model consist of the mean and standard deviation of each mixture components along with the weights. Mathematically, MDN for Gaussian family can be formulated as follows:

$$
p\left(\mathbf{y}_{i} \mid \mathbf{x}_{i}, \mathbf{w}\right)=\sum_{k=1}^{K} \alpha_{k} N\left(\mathbf{y}_{i} ; \mu_{k}, \sigma_{k}\right),
$$

where $x_{i} \in X$ are the input features, and $y_{i} \in Y$ are the outputs. Furthermore, $w$ correspond to the weights of the NN, $\mu_{k}$ and $\sigma_{k}$ are the parameters of the $K^{t h}$ distribution component along with the weights or mixing coefficients $\alpha_{k}$. During the training phase, the $\mathrm{NN}$ takes features $X$ as inputs and then tries to learn the mapping function to the parameters of mixture model $\left(\mu_{k}, \sigma_{k}\right.$ and $\left.\alpha_{k}\right)$ such that the components of the model captures the true underlying distribution $p(Y \mid X)$ as accurately as possible.

The MDN architecture used for path loss prediction is shown in Fig. 4. The 3D distance between UEs and BSs is used as the input feature of the NN. The $\mathrm{NN}$ has one input layer with 1 node, one hidden layer with 64 nodes having a rectified linear unit (ReLU) activation, and a output layer. The nodes of the output layer correspond to the number of mixture model parameters. For the path loss model, two Gaussian components are considered, one for LoS and another for NLoS, resulting in six parameters as the output nodes. Moreover, each set of output nodes has a different activation unit. The output node of the mixing coefficient must satisfy $\sum_{k=1}^{K} \alpha_{k}=1$. As a result, softmax activation is used. Similarly, the output nodes for $\sigma_{k}$ should provide positive values. Hence nonnegative exponential is used as an activation function. For

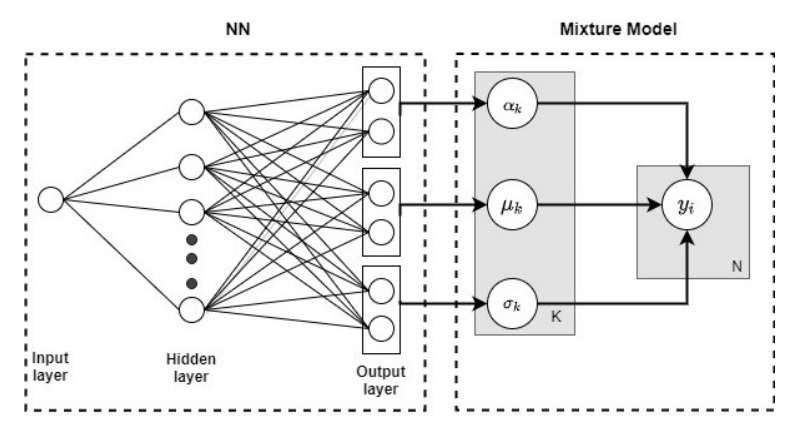

Fig. 4. MDN Architecture 
$\mu_{k}$, no activation is applied to the output nodes. The MDN model is implemented with tensor flow utilizing keras API. Moreover, negative log-likelihood is used as an error function, which is minimized with Adam optimizer [19]. The Adam optimizer have three hyper-parameters; $\alpha_{\text {adam }}$ (also known as the learning rate), $\beta_{1}$ and $\beta_{2}$. The value of $\beta_{1}$ and $\beta_{2}$ was selected to be 0.9 and 0.999 as per the suggestion of [19]. The learning rate was selected to be 0.01 after iterative testing.

To avoid over-fitting, regularization was implemented with the early stopping method [20]. For model training, a portion of the total data-set was selected, from which $20 \%$ of the data was used as a validation set for early stopping, and the remaining was used as a training data-set. The model was tested in the same way as the Bayesian one.

\section{COMPARISON OF THE PREDICTORS}

In this section, we analyze how the predictors perform when only a limited amount of data is available for training. As a reference, true path loss distributions are obtained by slicing the overall data-set (Fig. 1) into distance ranges. For example, the predicted distribution at $52 \mathrm{~m}$ distance is compared against the actual distribution obtained by considering path loss values of users within the range of $52 \mathrm{~m}$ to $57 \mathrm{~m}$.

To quantify prediction accuracy, we shall use the Kullback Leibler divergence, and the L1-norm, i.e. the Total Variation Distance, see [21]. The metrics also take into account that more frequent distances are more critical for model accuracy, and hence they get more weight.

KL divergence is a metric used to quantify how much information would be lost if one tries to approximate a true distribution with an approximate one. For the true distribution $p(x)$ and the approximate distribution $q(x)$ the KL divergence is defined as

$$
\mathrm{D}(p \| q)=\sum_{x \in X} p(x) \log \frac{p(x)}{q(x)} .
$$

The KL divergence takes values in $[0 ; \infty]$, with zero indicating that the two distributions are identical.

Moreover, TVD is another metric used for measuring the difference between two distributions. It is given by the $L_{1}$ norm and takes the values in $[0 ; 1]$ :

$$
\mathrm{d}_{\mathrm{tv}}=\frac{\sum_{x \in X}|p(x)-q(x)|}{2} .
$$

If two distributions are completely different then the TVD is 1 , while for two identical distributions the distance is 0 .

For each predictor, the above-mentioned metrics are estimated for the range of distances, and then the weighted average is calculated with the weights assigned based upon the UE geographical distribution in the scenario. For example, if there are 5K UEs at $200 \mathrm{~m}$ distance, then weight applied to the metric at this distance is $5 \mathrm{~K}$ divided by the total amount of users.

Furthermore, the predictors' capabilities are also tested in two set-ups, a network-wide and a site-specific. In the network-wide case, the predictors are trained using the data
TABLE II

NETWORK-WIDE VS CELL-SPECIFIC PREDICTORS

\begin{tabular}{cccccccccc}
\hline \multirow{2}{*}{ Metric } & \multicolumn{4}{c}{ MDN Predictor } & & \multicolumn{4}{c}{ Bayesian Predictor } \\
\cline { 2 - 4 } \cline { 7 - 10 } & $\mu_{\text {cell }}$ & $\sigma_{\text {cell }}$ & $\mu_{\text {Net }}$ & $\sigma_{\text {Net }}$ & & $\mu_{\text {cell }}$ & $\sigma_{\text {cell }}$ & $\mu_{\text {Net }}$ & $\sigma_{\text {Net }}$ \\
\hline KL divergence & 0.0071 & 0.0010 & 0.0082 & 0.0016 & & 0.0091 & 0.0005 & 0.0096 & 0.0018 \\
TVD & 0.0036 & 0.0003 & 0.0038 & 0.0004 & & 0.0040 & 0.0004 & 0.0041 & 0.0004 \\
\hline
\end{tabular}

collected from the entire network. Similarly, for site-specific scenario, model training is carried out on the data-sets of individual BS sites. The goal of this comparison is to learn if multiple site-specific predictors can perform better than when only one model is used for the entire network. In total, $10 \mathrm{~K}$ data points were used for the training. The results for KL divergence and TVD are summarised in Table II, where the mean and standard deviation of both metrics for all BS sites can be found. It can be seen that the site-specific approach gives better results than the network-wide for both predictors. Moreover, for all the models, the site-specific MDN predictor achieve the best prediction performance, and the network-wide Bayesian predictor has the worse accuracy.

Next, each predictor's capability is evaluated for different number of training data-set sizes. Fig. 5 and Fig. 6 show the performance of each model in terms of weighted $\mathrm{KL}$ divergence and TVD, respectively. As expected, increasing the size of data-set improves the prediction quality of the predictors. MDN predictor performs worse as compared to the Bayesian approach when a small data-set is available. However, it becomes better when trained on a larger number of data points. For both models, there is no noticeable improvement from the increase in the size of the data-set after a certain satisfactory prediction quality is achieved. This is logical, because, after a certain threshold, new data points are so close to each other that they do not provide additional information to the models.

Table III shows the comparison of the predictors with the

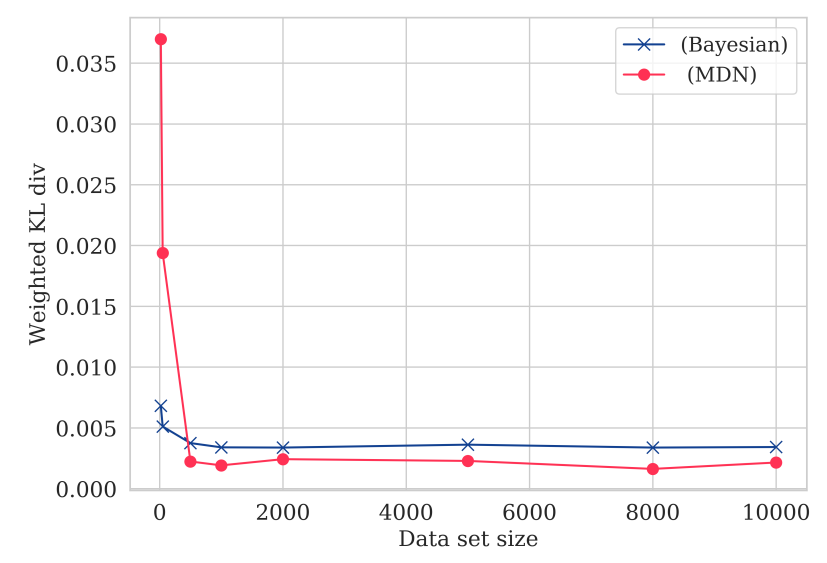

Fig. 5. Comparison of MDN and Bayesian predictors with respect to the size of the training data-set. Weighted KL divergence a metric. 


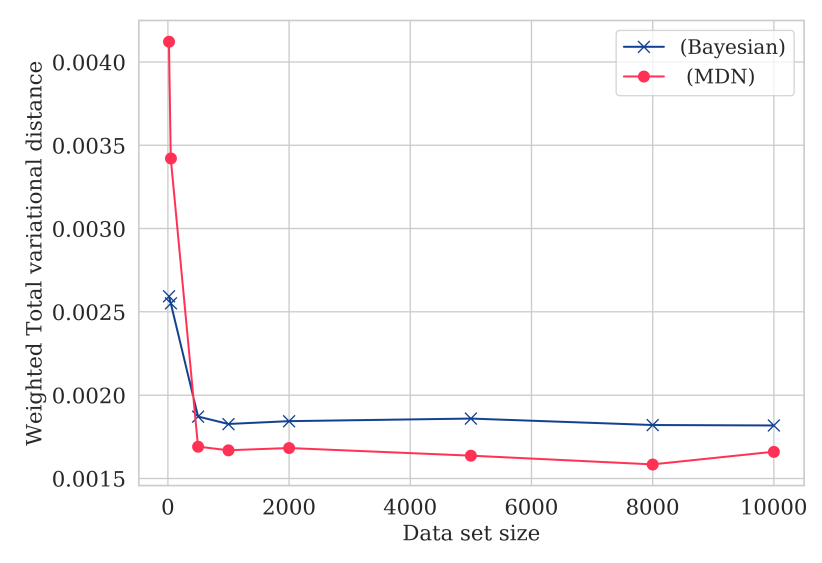

Fig. 6. Comparison of MDN and Bayesian predictors with respect to the size of the training data-set. Weighted TVD a metric.

TABLE III

PREDICTION QUALITY

\begin{tabular}{cccc}
\hline Metric & 3GPP Model & Bayesian Predictor & MDN Predictor \\
\hline KL divergence & 0.0435 & 0.0025 & 0.0018 \\
TVD & 0.0063 & 0.0015 & 0.0017 \\
\hline
\end{tabular}

standard 3GPP path loss model [9] as a reference. Here, the Bayesian and MDN network-wide predictors are trained with the largest data-set of $10 \mathrm{~K}$ points. The results show that without special fitting, the 3GPP model provides poor prediction capability in comparison to the considered predictors. Fig. 7 depicts the difference in the prediction of each model at a given distance around $55 \mathrm{~m}$. It can be seen that the distributions provided by the predictors overlap well with the true distribution. The 3GPP-based distribution has different shape and an additional offset.

\section{CONCLUSION}

In this study, two probabilistic path loss predictors for mmWave frequencies are developed. We use a hybrid approach. On the one hand, these predictors can incorporate domain knowledge, such as analytic formulae and the differ-

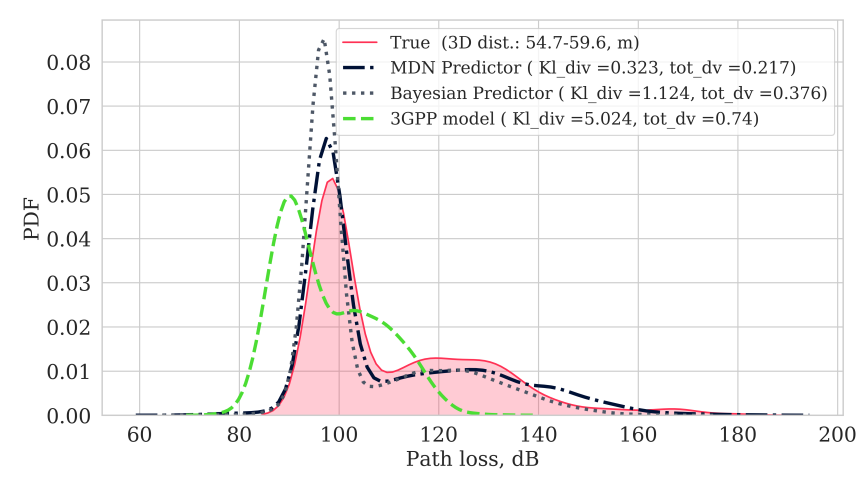

Fig. 7. True and predicted path loss distributions. ence between LoS and NLoS conditions. On the other hand, the predictors can be trained with available measurements and provide not only point estimates but the whole probabilistic distributions of the predicted values.

We demonstrate that the Bayesian approach is beneficial when there are only a few measurements available. However, the MDN predictor achieves higher accuracy on more massive data-sets. The predictors can also be customized additionally by using multiple site-specific data-sets.

In the paper, we train the predictors for the specific environment. In the future, the applicability of the models for the new scenarios different from the original one can be considered.

We expect that such predictors can be used as a part of more complex scenario-specific end-to-end network performance evaluation models and for network planing tasks.

\section{REFERENCES}

[1] ETSI GS ZSM, "Zero-touch network and Service Management (ZSM); Reference Architecture," Tech. Rep., 2019.

[2] M. R. Akdeniz, Y. Liu et al., "Millimeter wave channel modeling and cellular capacity evaluation," IEEE Journal on Selected Areas in Communications, vol. 32, no. 6, pp. 1164-1179, 2014.

[3] J. M. Kelif, M. Coupechoux, and M. Mansanarez, "A 3D beamforming analytical model for 5G wireless networks," 14th International Symposium on Modeling and Optimization in Mobile, Ad Hoc, and Wireless Networks, WiOpt 2016.

[4] T. Bonald, S. E. Elayoubi, and Y. T. Lin, "A flow-level performance model for mobile networks carrying adaptive streaming traffic," IEEE GLOBECOM, 2015.

[5] A.-R. Al-Saedy, Murtadha et al. "Coverage and effective capacity in downlink MIMO multicell networks with power control," IEEE Access, vol. 6, pp. 9173-9185, 2018.

[6] T. S. Rappaport, Y. Xing et al., "Overview of Millimeter Wave Communications for a Focus on Propagation Models," IEEE Transactions on Antennas and Propagation, vol. 65, no. 12, pp. 6213-6230, 2017.

[7] S. Sun, T. S. Rappaport et al., "Investigation of Prediction Accuracy, Sensitivity, and Parameter Stability of Large-Scale Propagation Path Loss Models for 5G Wireless Communications," IEEE Transactions on Vehicular Technology, vol. 65, no. 5, pp. 2843-2860, 2016.

[8] S. Sun, , T. A. Thomas et al., "Path loss, shadow fading, and line-ofsight probability models for $5 \mathrm{~g}$ urban macro-cellular scenarios," in IEEE GLOBECOM Workshops (GC Wkshps), 2015, pp. 1-7.

[9] 3GPP, "Study on channel model for frequencies from 0.5 to $100 \mathrm{ghz}$ (release 15)," Tech. Rep., 2018.

[10] R. D. Timoteo, D. C. Cunha, and G. D. Cavalcanti, "A proposal for path loss prediction in urban environments using support vector regression," in Proc. Advanced Int. Conf. Telecommun, 2014, pp. 1-5.

[11] M. Ribero, R. W. Heath et al., "Deep learning propagation models over irregular terrain," in IEEE ICASSP, 2019, pp. 4519-4523.

[12] M. K. Samimi, T. S. Rappaport, and G. R. Maccartney, "Probabilistic Omnidirectional Path Loss Models for Millimeter-Wave Outdoor Communications," IEEE WCL, vol. 4, no. 4, pp. 357-360, 2015.

[13] C. Davidson-Pilon, Bayesian methods for hackers: probabilistic programming and Bayesian inference, 2015.

[14] TensorFlow Probability (TFP) library. [Online]. Available: https: //www.tensorflow.org/probability/

[15] C. Andrieu and J. Thoms, "A tutorial on adaptive MCMC," Statistics and computing, vol. 18, no. 4, pp. 343-373, 2008.

[16] A. Gelman, Carlin et al., "Bayesian data analysis." CRC press, 2013, ch. 6, p. 143.

[17] C. M. Bishop, "Mixture density networks," 1994.

[18] - Pattern recognition and machine learning. Springer, 2006.

[19] D. P. Kingma and J. Ba. (2014) Adam: A method for stochastic optimization.

[20] L. Prechelt, "Early stopping-but when?" in Neural Networks: Tricks of the trade. Springer, 1998.

[21] "M. Lovric, Ed." in International Encyclopedia of Statistical Science. Springer Berlin Heidelberg, 2011. 